\title{
Towards Flexible Remote Therapeutic Interventions
}

\author{
Marc Schickler ${ }^{1}$, Rüdiger Pryss ${ }^{1}$, Johannes Schobel ${ }^{1}$, Winfried Schlee ${ }^{2}$, Thomas Probst ${ }^{1}$, Manfred Reichert $^{1}$ \\ ${ }^{1}$ Institute of Databases and Information Systems, Ulm University, Germany \\ ${ }^{2}$ Clinic and Policlinic for Psychiatry and Psychotherapy, University of Regensburg, Germany \\ ${ }^{1}\{$ marc.schickler, ruediger.pryss, johannes.schobel, thomas.probst, manfred.reichert\}@uni-ulm.de \\ winfried.schlee@gmail.com
}

\begin{abstract}
In the context of therapeutic interventions, smart mobile devices are becoming increasingly important. First, they can properly assist patients in performing their homework - a support required for more efficient therapeutic interventions. Second, mobile applications enable therapists to monitor homework outcomes. From a technical perspective, frequently required changes of the mobile applications supporting therapeutic interventions constitute a major challenge. To tackle the latter for a multitude of remote therapeutic interventions, e.g., in psychotherapy or physiotherapy, we deploy process management technology to smart mobile devices. This paper discusses flexibility issues addressed by the mobile processes. Particularly, the achieved flexibility, in turn, increases the practical benefits of smart mobile devices in the context of remote therapeutic interventions.
\end{abstract}

Keywords-Mobile service, flexible therapeutic intervention support, mobile context, mobile process, mobile assistance.

\section{INTRODUCTION}

Clinical studies have revealed that therapeutic interventions constitute a fundamental part of most therapies. The variety of therapeutic interventions, in turn, ranges from simple medication intakes up to complex homework, assigned to the patient by a therapist. Taking the diversity of homework assignments into account, therapists crave for a flexible IT support. Existing approaches often neglect the use of smart mobile devices and often focus on specifically tailored therapeutic intervention tools. Consequently, if therapists want to adjust a therapeutic intervention, costly and time-consuming changes of these tools become necessary. To remedy these requirements, we developed an approach, which relies on process management technology in order to flexibly cope with remote therapeutic interventions based on mobile processes. In practice, therapeutic interventions consist of homework (e.g., daily back exercises) that, in turn, comprises several activities. For example, in the context of a daily back exercise, three activities need to be performed. Mobile processes, in turn, constitute the basis for dealing with both homework and exercise activities. Furthermore, mobile processes may capture the context (e.g., being in the kitchen), in which exercises shall be performed. Finally, they ease the integration of smart mobile devices sensors [1].

Section II discusses how mobile processes foster flexible therapeutic interventions. Section III presents related work, whereas Section IV concludes the paper.

\section{Flexible Therapeutic Intervention Support}

When developing our approach, we aimed at reducing the complexity of configuring and adjusting therapeutic interventions by domain experts. The configurator we developed, therefore, adopts concepts from end-user development, which have proven feasibility [2]. Particularly, the developed approach shall address flexibility issues in order to meet practical demands. This section discusses these issues along the three major lifecycle phases (cf. Fig. 2):

(1) Design time flexibility is enabled through the configurator. Thereby, two concepts contribute to the flexible support of therapeutic interventions. First, we allow for the exchange of exercise activities based on configurable subprocesses, e.g., in Fig. 1 activity effort may be exchanged using a sub-process. The contextual information required to evaluate whether biking or running shall be configured for this sub-process is specified by therapists using the configurator. Second, the way therapeutic interventions are mapped to mobile processes enables us to assist therapists in creating executable mobile processes. Therefore, the configurator assists therapists when modeling homework as well as the exercise activities and their interdependencies.

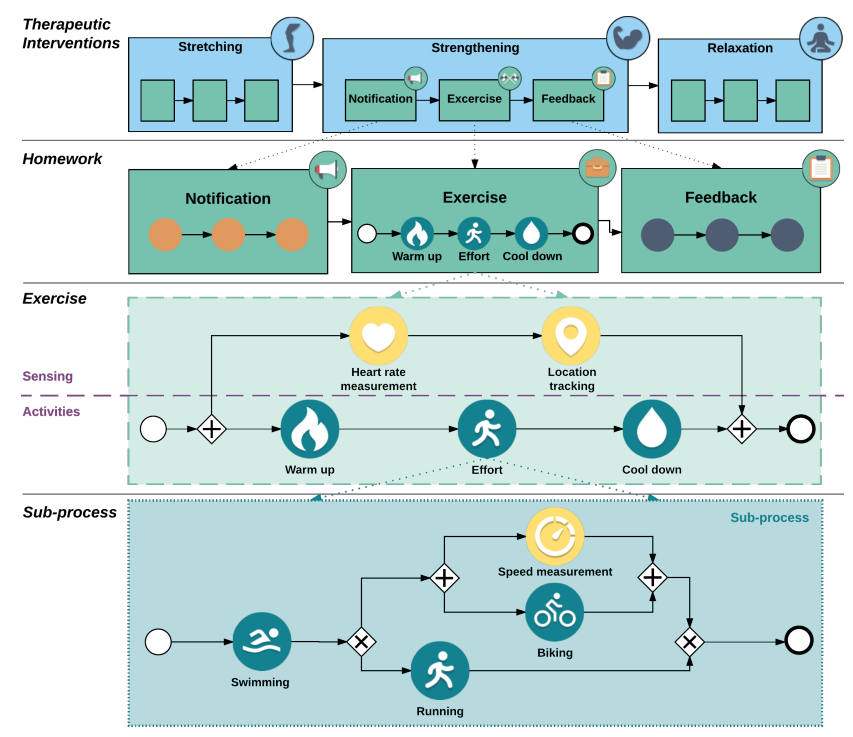

Figure 1. Mobile Process 


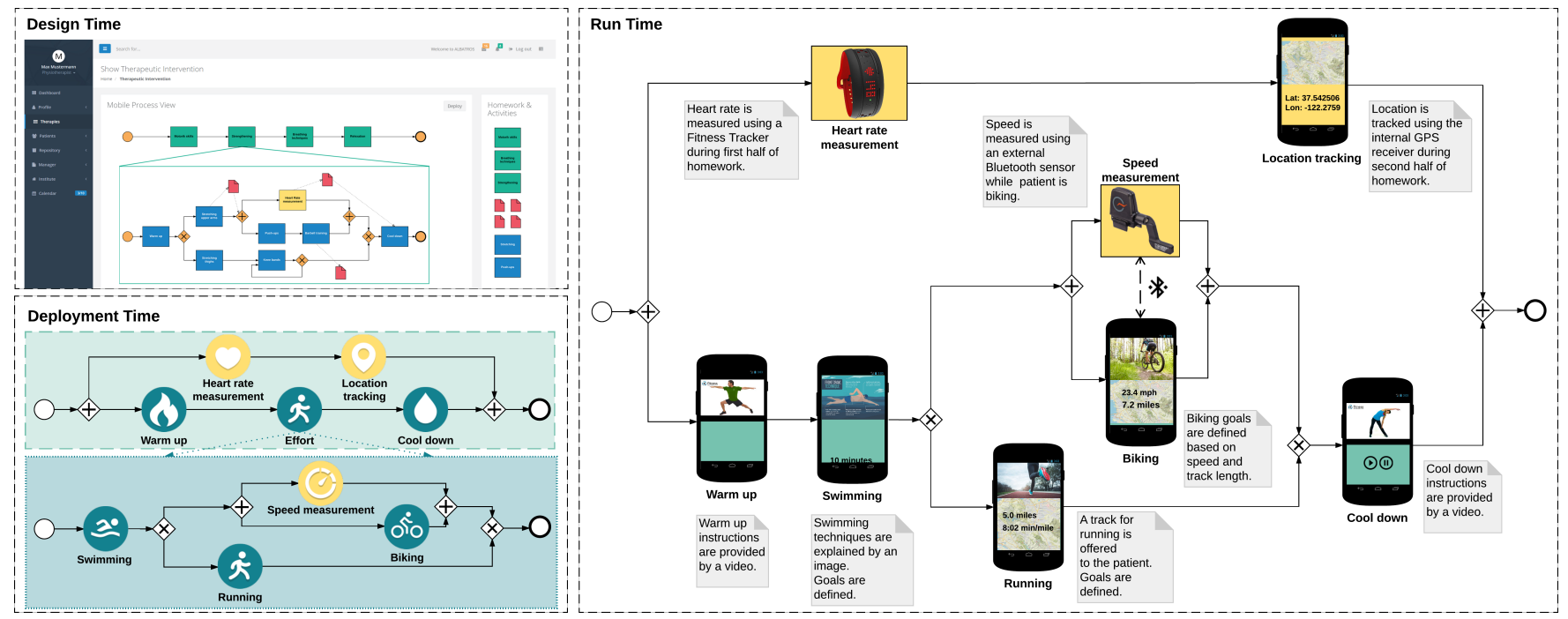

Figure 2. Providing Flexibility in the Lifecycle of Remote Therapeutic Interventions

(2) Deployment time. The designed therapeutic intervention is mapped to a process model, which is then deployed to the respective smart mobile device(s). In order to enable a flexible and robust execution of the therapeutic intervention procedure on the smart mobile device, mobile process management technology is applied [1].

(3) Run time flexibility addresses various issues. First, a process engine is run on the smart mobile device. It enables the robust execution of the therapeutic intervention process model defining its logic, i.e., the process engine enacts and executes the respective process model. In turn, a particular process activity (e.g., showing an instruction video) and its corresponding user interface is realized using executable components [1]. The process engine dynamically loads the components needed for an application scenario during run time. Second, a sensor framework allows connecting sensors (e.g., heart rate) with the smart mobile device. Third, mobile processes enable us to monitor therapeutic interventions.

\section{RELATED WORK}

Several approaches deal with the IT support for therapeutic interventions in the context of patient treatments. In particular, they target at the support of psychotherapies [3], [4]. Recent works, e.g., [5], have revealed that mobile applications can be used to properly perform therapeutic interventions. In addition, there exist IT-driven approaches that may be configured by domain experts to increase the efficacy of a psychotherapy [6]. However, none of these approaches aims at a generic technical solution covering various therapeutic interventions (i.e., not only psychotherapy) and using smart mobil devices.

\section{Summary AND OUTLOOK}

Smart mobile devices offer promising perspectives for remote therapeutic interventions. This paper introduced the concept of mobile processes, which enables flexibility along the lifecycle phases of remote theraupeutic interventions. Our configurator indicates that the complexity to configure and adjust therapeutic interventions can be reduced. In this context, an evaluation study is currently designed to improve the usability of the developed configurator. Moreover, patients benefit from personalized therapeutic interventions running on smart mobile devices. Altogether, the support of therapeutic interventions on smart mobile devices constitutes an important step towards more effective patient therapies.

\section{REFERENCES}

[1] J. Schobel, R. Pryss, M. Schickler, and M. Reichert, "A lightweight process engine for enabling advanced mobile applications," in 24th Int'l Conf. on Cooperative Information Systems. Springer, 2016, pp. 552-569.

[2] _ , "A Configurator Component for End-User Defined Mobile Data Collection Processes," in Proc 14th Int'l Conf on Service Oriented Computing, October 2016.

[3] D. J. Cox, D. A. Tisdelle, and J. Culbert, "Increasing adherence to behavioral homework assignments," Journal of behavioral medicine, vol. 11, no. 5, pp. 519-522, 1988.

[4] E. Kuhn, B. Weiss, K. Taylor, J. Hoffman, K. Ramsey, R. Manber, P. Gehrman, J. Crowley, J. Ruzek, and M. Trockel, "Cbti coach: a description and clinician perceptions of a mobile app for cognitive behavioral therapy for insomnia," Journal of clinical sleep medicine, vol. 12, no. 4, p. 597, 2016.

[5] A. Aguilera and F. Muench, "There's an app for that: Information technology applications for cognitive behavioral practitioners," The Behavior therapist/AABT, vol. 35, no. 4, p. $65,2012$.

[6] S. Watts, A. Mackenzie, C. Thomas, A. Griskaitis, L. Mewton, A. Williams, and G. Andrews, "Cbt for depression: a pilot rct comparing mobile phone vs. computer," BMC psychiatry, vol. 13, no. 1, p. 49, 2013. 